\title{
Plasmodium falciparum variant erythrocyte surface antigens: a pilot study of antibody acquisition in recurrent natural infections
}

Elise Schieck ${ }^{1,2,10^{*}}$ D, E. Jane Poole ${ }^{3}$, Anja Rippert ${ }^{2,4}$, Judy Peshu' ${ }^{1}$, Philip Sasi ${ }^{5}$, Steffen Borrmann ${ }^{1,6,7 \dagger}$ and Peter C. Bull ${ }^{1,8,9 \dagger}$

\begin{abstract}
Background: During intra-erythrocytic replication Plasmodium falciparum escapes the human host immune system by switching expression of variant surface antigens (VSA). Piecemeal acquisition of variant specific antibody responses to these antigens as a result of exposure to multiple re-infections has been proposed to play a role in acquisition of naturally acquired immunity.

Methods: Immunofluorescence was used to explore the dynamics of anti-VSA IgG responses generated by children to (i) primary malaria episodes and (ii) recurrent P. falciparum infections.

Results: Consistent with previous studies on anti-VSA responses, sera from each child taken at the time of recovery from their respective primary infection tended to recognize their own secondary parasites poorly. Additionally, compared to patients with reinfections by parasites of new merozoite surface protein 2 (MSP2) genotypes, baseline sera sampled from patients with persistent infections (recrudescence) tended to have higher recognition of heterologous parasites. This is consistent with the prediction that anti-VSA IgG responses may play a role in promoting chronic asymptomatic infections.
\end{abstract}

Conclusions: This pilot study validates the utility of recurrent natural malaria infections as a functional readout for examining the incremental acquisition of immunity to malaria.

Keywords: Plasmodium falciparum, Malaria, Recurrent infection, Recrudescent infection, Antibody aquisition

\section{Background}

Children who grow up in malaria endemic areas develop naturally acquired immunity to severe forms of the disease. This immunity takes several years to build up, even in hyperendemic areas, and probably never confers sterile immunity. Thus, many individuals in malarious areas carry asymptomatic infections. The transition from malaria to asymptomatic infection is still poorly

\footnotetext{
*Correspondence: e.schieck@cgiar.org

†'Steffen Borrmann and Peter C. Bull contributed equally to this work

${ }^{10}$ Present Address: International Livestock Research Institute, Old

Naivasha Road, P.O. Box 30709, Nairobi 00100, Kenya

Full list of author information is available at the end of the article
}

understood. The staggered age distribution of severe malaria, mild malaria and asymptomatic infection suggests distinctive immunological mechanisms that (i) confer protection against pathogenic features of infection (ii) limit parasite replication leading to control of parasite burden. The role of antibodies is demonstrated through studies in which purified IgG from immune adults was used to bring about rapid reduction in parasitaemia and symptoms of malaria $[1,2]$. One novel way to explore this transition further is through observation of recrudescences, which can occur when drug treatment fails to eliminate a blood stage infection [3].

A major target for protective antibodies against malaria are the variant surface antigens (VSA) that are anchored 
in the cell membrane of infected erythrocytes. These antigens mediate adhesive parasite-host interactions thought to be essential for evading innate immune clearance mechanisms in the spleen. Variant specific anti-VSA immune responses are thought to play an important role in the development of naturally acquired immunity to malaria [4-6]. Following an episode of malaria individuals develop anti-VSA antibodies that are highly specific to the isolate that caused that episode of malaria (henceforth termed homologous parasites) [4-8]. Carriage of anti-VSA antibodies imposes a selection pressure on the infecting blood stage parasite population restricting the diversity of VSAs expressed $[4,5,9]$. More directly an in vivo switch in VSA expression has been observed with accompanying acquisition of antibodies specific to the respective VSAs [10].

Plasmodium falciparum Erythrocyte Membrane Protein 1 (PfEMP1), encoded by the large multigene var family is thought to be the major VSA [11]. Each haploid genome contains about 60 var genes, and expression is mutually exclusive at the individual parasite level (reviewed in [12]). Immune selection of PfEMP1 expression by host antibodies has been shown indirectly by correlation of expression of specific subsets of var genes with age $[13,14]$, and by negative association with antiVSA antibody repertoire [14-16].

Here, to explore the transition from malaria disease to asymptomatic infection and the relationship between recrudescence and immunity to VSA, anti-VSA antibody acquisition was studied in children within the age bracket where the transition from symptomatic to asymptomatic infections occurs, who presented to hospital with mild malaria, and who returned within 84 days with recurrent parasitaemia. The kinetics of antibody acquisition to the baseline and recurrent isolates was studied. The anti-VSA antibody repertoire of sera from patients that carried parasites that recrudesced was compared to the repertoire of those that experienced reinfection by new genotypes only.

\section{Methods}

\section{Origin and storage of samples}

In this study, samples from children presenting with mild malaria who were included in a Phase III, randomized, non-inferiority trial was used to assess the efficacy and safety of dihydroartemisinin-piperaquine (Artekin) in comparison with artemether-lumefantrine (Coartem $\odot$ ) in Pingilikani, Kenya, with a follow up period of 84 days. This study has been described in detail elsewhere [17]. Children were enrolled if presenting with uncomplicated malaria and fulfilling all criteria as described [17]. They were monitored for 3 days on-site during the administration of therapy and the mother/guardian was asked to return with the child for scheduled visits on days 7,14 , $21,28,35,42$ and 84 post-enrollment, or if any symptoms occurred. During this follow up period, samples (serum, blood samples for gDNA extraction and live P. falciparum isolates) were collected at any time the children were parasitaemic according to microscopic examination as described [17]. Serum was additionally collected on days 3, 7, 14, 28 and 42. All samples were anonymized. Serum was stored at $-80{ }^{\circ} \mathrm{C}$. Full blood containing parasitized red blood cells was collected and mixed with cryosolution (28\% glycerol, 3\% (w/v) sorbitol, $0.64 \%$ saline) at a volumetric ratio of about 1:4 (packed cell volume:cryosolution) and stored in liquid nitrogen.

\section{Description of isolates used}

During the follow up period recurrent parasitaemias were screened for recrudescences using MSP2 genotyping. Initial screening was done using the RFLP method described by Felger et al. [18] and isolates from 9 patients were selected for the study described here if the patients harboured a recrudescence within the first 42 days of follow up and if the parasitaemia was $>0.1 \%$ (Tables 1 and 2). Flow cytometry data was obtained from 7 of these patients, and out of these, 6 patients could later be confirmed as harbouring recrudescence by a superior resolution capillary electrophoresis MSP2 genotyping method [19] using oligo N5 with JOE instead of VIC, on the ABI genetic analyzer 3130 (Additional file 1: Table S1). However, it cannot be excluded that this will include false recrudescences by infections caused by parasites containing the same MSP2 alleles.

\section{Description of sera used}

Sera from these 7 patients, taken at day of recruitment and at follow-up visits (days 0, 3, 7, 14, 28, 42 and 84, and when patient visited clinic with parasitaemia during the follow-up period), were used to study antibody acquisition towards the respective infecting parasites in each of these patients over time. Whenever a serum is tested against an isolate from the same patient, they are referred to as homologous serum and isolate.

To test heterologous recognition of the isolates, i.e., testing serum derived from different patients than the

Table 1 Sample (parasite isolate) characteristics

\begin{tabular}{ll}
\hline & Mean \pm SD \\
\hline Age of patient (at inclusion in study) & 32.9 months \pm 9.9 \\
Time to first recurrence & 35 days \pm 7.0 \\
Number of MSP2 alleles (baseline infections) & $2.29 \pm 0.95$ \\
Number of MSP2 alleles (recurrent infections) & $2.25 \pm 2.01$ \\
Parasitaemia (baseline infections) & $3.3 \% \pm 2.3$ \\
Parasitaemia (recurrent infections) & $1.4 \% \pm 1.9$
\end{tabular}


Table 2 Parasite isolates included in the study

\begin{tabular}{|c|c|c|c|c|c|c|c|c|}
\hline Patients & $\begin{array}{l}\text { Female }(\mathbf{f}) / \\
\text { male }(\mathrm{m})\end{array}$ & $\begin{array}{l}\text { Age } \\
\text { (months) }\end{array}$ & $\begin{array}{l}\text { Blood } \\
\text { group }\end{array}$ & $\begin{array}{l}\text { Day of parasit- } \\
\text { aemia }\end{array}$ & $\begin{array}{l}\text { Parasitaemia } \\
\text { (\%) }\end{array}$ & $\begin{array}{l}\text { Flow } \\
\text { cytometry } \\
\text { analysis }\end{array}$ & $\begin{array}{l}\text { Asymptomatic } \\
\text { (tympanic } \\
\text { tempera- } \\
\text { ture }<37.5^{\circ} \mathrm{C} \text { ) }\end{array}$ & $\begin{array}{l}\text { No of infecting } \\
\text { clones }^{\mathrm{a}}\end{array}$ \\
\hline \multirow[t]{3}{*}{119} & $\mathrm{~F}$ & 41 & A & 0 & 6.25 & Yes & & 2 \\
\hline & & & & 35 & 0.68 & Yes & No & 2 \\
\hline & & & & 84 & 2.19 & No & No & 1 \\
\hline \multirow[t]{2}{*}{150} & M & 30 & A & 0 & 1.69 & No & & 3 \\
\hline & & & & 42 & 6.09 & Yes & No & 2 \\
\hline \multirow[t]{3}{*}{171} & M & 13 & $A B$ & 0 & 2.57 & Yes & & 3 \\
\hline & & & & 21 & 0.07 & Yes & Yes & 1 \\
\hline & & & & 76 & 2.06 & Yes & No & 2 \\
\hline \multirow[t]{2}{*}{178} & M & 35 & A & 0 & 2.71 & Yes & & 6 \\
\hline & & & & 42 & 4.11 & Yes & No & 1 \\
\hline \multirow[t]{3}{*}{221} & M & 38 & A & 0 & 6.76 & Yes & & 7 \\
\hline & & & & 35 & 0.27 & No & Yes & 4 \\
\hline & & & & 78 & 0.14 & No & Yes & 7 \\
\hline \multirow[t]{2}{*}{233} & M & 42 & 0 & 0 & 2.22 & Yes & & 3 \\
\hline & & & & 35 & 0.47 & Yes & Yes & 7 \\
\hline \multirow[t]{4}{*}{245} & $\mathrm{~F}$ & 31 & 0 & 0 & 1.01 & Yes & & 4 \\
\hline & & & & 35 & 0.11 & No & Yes & 6 \\
\hline & & & & 63 & 0.67 & Yes & Yes & 2 \\
\hline & & & & 84 & 0.11 & Yes & Yes & 9 \\
\hline
\end{tabular}

${ }^{a}$ According to MSP2 analysis (capillary electrophoresis)

parasite isolate tested, a panel of heterologous sera (Table 3) from 14 (blood group A) and 4 (blood group $\mathrm{AB}$ ) patients were chosen. The panel of 4 sera (blood group $\mathrm{AB}$ ) was used to test parasites from patient 171 , the only patient with blood group $A B$ (Table 2). All sera originated from the same study described above, from children who suffered from recurrent parasitaemias within the follow up period. Four of the sera in the panel originated from patients whose isolates were further characterized in this study $(119,150,178$ and 221), but when the serum-isolate pair (derived from the same patient) were tested this was recorded as homologous testing, not heterologous testing. From all 18 patients of the heterologous serum panel, sera from day 0 (acute) and 14 (convalescent) were tested.

Improved MSP2 analysis using capillary electrophoresis [19] was performed as described above on the isolates derived from the patients whose sera were included in the heterologous serum panel to differentiate between sera derived from patients who experienced recrudescences and reinfections during the follow up period.

\section{Flow cytometry}

Live isolates were removed from liquid nitrogen, washed with ice cold $3.5 \% \mathrm{NaCl}$, then washed with culture medium (RPMI 1640; Gibco), and transferred into culture flasks where the isolates were allowed to stabilize for 18-24 h under standard culture conditions [20] until trophozoite stage and were then analysed using flow cytometry. The procedure of freezing and thawing of the already small blood sample volumes originating from young children reduces the volume further mainly due to red blood cell lysis. For each test, $0.5 \mu \mathrm{l}$ of the isolate pellet were incubated $30 \mathrm{~min}$ in $40 \mu \mathrm{l}$ of PBS $(0.1 \% \mathrm{BSA})$ with $0.1 \mathrm{mg} / \mathrm{ml}$ Ethidium Bromide (Sigma) and $1 \mu \mathrm{l}$ serum. Negative controls were no serum and non-immune pooled blood group AB serum from non-exposed European adults, and a positive control, hyperimmune blood group $A B$ adult serum from the Kilifi area also used in previous studies $[14,21]$ was included. After washing (with PBS, centrifuging at $900 \mathrm{~g}$ for $5 \mathrm{~min}$ ) cells were incubated in $30 \mu \mathrm{l}$ of PBS (0.1\% BSA) containing antihuman-CD45-PC7 (1:30 v/v) (IM3548; Beckman Coulter), antihuman IgG-FITC (1:1 v/v) (FA004; the Binding Site) and antihuman IgM-SPRD (1:30 v/v) (735986; Beckman Coulter). After washing three times (with PBS, centrifuging at $900 \mathrm{~g}$ for $5 \mathrm{~min}$ ) samples were resuspended in PBS (0.1\% BSA) and analysed using an FC500 Flow Cytometer (Beckman Coulter).

FlowJo and Excel were used for the initial flow cytometry analysis, where first nucleus containing cells stained by ethidium bromide were included, i.e. leukocytes and 
Table 3 Heterologous serum panel

\begin{tabular}{|c|c|c|c|c|c|c|c|c|c|}
\hline \multirow[t]{2}{*}{ Patients } & \multirow{2}{*}{$\begin{array}{l}\text { Male }(m) / \\
\text { female (f) }\end{array}$} & \multirow{2}{*}{$\begin{array}{l}\text { Age } \\
\text { (months) }\end{array}$} & \multirow{2}{*}{$\begin{array}{l}\text { Blood } \\
\text { group }\end{array}$} & \multirow{2}{*}{$\begin{array}{l}\text { Para- } \\
\text { sitemia } \\
\text { baseline }\end{array}$} & \multirow{2}{*}{$\begin{array}{l}\text { Day of recur- } \\
\text { rence }\end{array}$} & \multirow{2}{*}{$\begin{array}{l}\text { Recurrent infec- } \\
\text { tion asymptomatic } \\
\text { (tympanic tempera- } \\
\text { ture }<37.5^{\circ} \mathrm{C} \text { ) }\end{array}$} & \multirow{2}{*}{$\begin{array}{l}\text { Recrudescent } \\
\text { infections }^{a}\end{array}$} & \multicolumn{2}{|c|}{ Number of infecting clones ${ }^{a}$} \\
\hline & & & & & & & & $\begin{array}{l}\text { Baseline } \\
\text { infec- } \\
\text { tions }\end{array}$ & $\begin{array}{l}\text { Recurrent infections } \\
\text { (recrudescent/new } \\
\text { infections) }{ }^{\mathrm{a}}\end{array}$ \\
\hline 56 & $M$ & 44 & $A B$ & 0.10 & 49 & Yes & & 1 & $0 / 1$ \\
\hline 77 & $M$ & 34 & A & 0.08 & 35 & No & Yes & 5 & $3 / 3$ \\
\hline 105 & $M$ & 43 & $A B$ & 1.29 & 49 & Yes & & 1 & $0 / 1$ \\
\hline 111 & $M$ & 36 & A & 0.11 & 28 & Yes & & 1 & $0 / 1$ \\
\hline 115 & & & $A B$ & 1.5 & 49 & Yes & & 1 & $0 / 3$ \\
\hline 119 & $\mathrm{~F}$ & 41 & A & 6.25 & 35 & No & Yes & 2 & $1 / 1$ \\
\hline 127 & $M$ & 46 & A & 0.85 & 45 & Yes & & 5 & $0 / 1$ \\
\hline 144 & $M$ & 48 & $A$ & 5.05 & 40 & No & & 2 & $0 / 1$ \\
\hline 150 & $M$ & 30 & A & 1.69 & 42 & No & & 3 & $0 / 2$ \\
\hline 160 & $\mathrm{~F}$ & 10 & A & 0.56 & 42 & No & & 1 & $0 / 4$ \\
\hline 162 & $\mathrm{~F}$ & 25 & A & 0.06 & 49 & No & Yes & 2 & $1 / 1$ \\
\hline 169 & $M$ & 33 & $A$ & 5.38 & 42 & Yes & & 2 & $0 / 2$ \\
\hline 178 & $M$ & 35 & $A$ & 2.71 & 42 & No & Yes & 6 & $1 / 0$ \\
\hline 181 & $F$ & 42 & $A$ & 0.05 & 42 & Yes & Yes & 4 & $4 / 0$ \\
\hline 191 & $\mathrm{~F}$ & 44 & $A$ & 3.99 & 54 & Yes & & 1 & $0 / 2$ \\
\hline 221 & $M$ & 38 & $A$ & 6.75 & 35 & Yes & Yes & 7 & $2 / 2$ \\
\hline 246 & $M$ & 40 & A & 0.27 & 40 & No & & 1 & $0 / 10$ \\
\hline 247 & $M$ & 32 & $A B$ & 0.47 & 56 & No & & 5 & $0 / 4$ \\
\hline
\end{tabular}

Sera from day 0 (acute) and day 14 (convalescent) was used from all patients

a According to MSP2 analysis (capillary electrophoresis)

infected red blood cells (irbcs) at the trophozoite stage. At this stage ring stage parasites were excluded by low ethidium bromide staining. Next CD45-containing leukocytes were excluded and finally the IgG recognition measured as mean fluorescence intensity (MFI).

The flow cytometer was set to count 1000 irbcs. Samples where less than 200 irbcs (due to small sample volumes and low parasitaemia) had been measured were excluded from analysis. When the volume of cells was smaller than expected (pipetting errors on semi-packed cell pellets), the last cells measured would give unusually many counts with high MFI values. To exclude these erroneous measurements, samples where the whole population of measured cells (mostly noninfected red blood cells) had more than $2.5 \%$ IgG positive cells, defined by a cut-off at MFI arbitrary units of 12, were excluded from analysis. Manual analysis confirmed that no samples with high parasitaemia were excluded by this process. All flow cytometry results are presented in this study as the MFI for each isolate-serum pair, with the individual "no serum" sample MFI subtracted. The "no serum" value for each parasite isolate was subtracted rather than the value of the negative control pool to allow comparison of the data with the negative control group. The MFI values for each measurement together with parasite isolate and serum information is presented in the Additional file 1: Table S1.

If the no serum sample was missing (excluded by reasons explained above) or differing by more than $90 \%$ from the median of all no serum samples, the whole series, i.e. all data from the corresponding isolate, was excluded.

To ensure that the measured fluorescence was not simply an effect of higher parasitaemia in the sample, i.e. an experimental artefact, linear regression analysis was performed for fluorescence vs. parasitaemia but no correlation was found ( $\mathrm{P}=0.27$, not shown).

All flow cytometry results used for analysis is presented in Additional file 1: Table S1.

\section{Statistical analysis}

Graphpad Prism 6.0e for MAC OS X and Genstat ${ }^{\circledR}$ 16th Edition were used for analysis and graphical presentation. A mixed-effects linear regression model using a REstricted Maximum Likelihood (REML) [22] approach was used in each analysis described below. In all analyses below, random effects for patient and serum were used to allow for correlation in reactivity of sera and isolated parasites originating from the same patient.

To test for intrinsic differences in the ability of the parasites to be recognized, the data were initially transformed 
by natural logarithm, $\log _{\mathrm{e}}(\mathrm{MFI}+0.145)$ to make the smallest observation positive and to normalize the distribution. Fixed effects for response type (originating from a patient that responded to its parasite isolate vs. a patient that did not) and serum day (day 0 or 14), together with their interaction were then tested in the model.

The same analysis was performed to test for a difference between recognition of baseline vs. recurrent isolates. In this case the fixed effects were isolate type (baseline or recurrent) and serum day (day 0 or 14).

The ability of homologous sera taken before recurrence to recognize the recurrent parasites was also compared to that of their overall recognition by heterologous sera with a linear mixed effect regression model. In this analysis, only sera taken before the recurrent infection were used. The data were transformed by $\log _{\mathrm{e}}(\mathrm{MFI}+0.062)$ to make the smallest observation positive. Fixed effects tested in the model were serum type (homologous recognition of recurrent isolates vs. recognition of the same recurrent isolates by heterologous sera) and serum day (day 0 or 14).

To compare the anti-VSA antibody repertoire of children who eliminated the baseline parasites to that of those children who did not, a similar linear mixed effect regression model was used. The data were transformed by $\log _{\mathrm{e}}(\mathrm{MFI}+0.145)$ to make the smallest observation positive. Fixed effects for serum type (originating from a patient that eliminated its parasite infection vs. a patient that did not) and serum day (day 0 or 14) were fitted.

Histograms or residuals, fitted-value plots and normal plots were performed to test the suitability of the transformed data in the linear mixed effect regression models.

\section{Results}

\section{Characterization of parasite isolates}

For this study, samples from children presenting with mild malaria who were included in a dihydroartemisininpiperaquine vs. artemether-lumefantrine drug trial were used [17]. In total 14 parasite isolates from 7 of these patients were analysed for antibody recognition (Tables 1,2). All the children involved are within the age bracket where Plasmodium infections transit from causing disease to being asymptomatic. Table 2 also shows whether the recurrent infections were asymptomatic (tympanic temperature $<37.5$ ) at the time of detection.

\section{Characterization of sera}

Sera derived from children in the same study were used to test antibody recognition of the isolates described above. Homologous sera (sera derived from the same patient as the parasite isolate tested) were used to test acquisition of antibodies to the infecting isolates of the respective patients. Sera from all available time points during the study follow up were used.
Heterologous sera (sera derived from other patients than the tested isolate) were used to test general recognition of the isolates in the population. For this a panel of sera from in total 18 patients in the same study were chosen to characterize heterologous recognition of the parasite isolates (Table 3). Sera from day 0 (acute) and 14 (convalescent) were used. All the patients contributing serum to this panel had recurrent infections during the follow-up period of 84 days, and Table 3 shows the day of recurrence and whether the detected recurrent infection was asymptomatic at the time of detection. Active weekly follow-up as in this study is likely to detect an increased number of asymptomatic infections, here $>50 \%$ of the recurrent infections were asymptomatic.

\section{Antibody acquisition to infecting isolates varies between patients}

Antibodies to the infected red blood cell (irbc) surface were measured by immunofluorescence and flow cytometry. To explore the kinetics of antibody responses to irbc sampled at baseline (day 0 ) and at the time of recurrent parasite infections each parasite isolate was tested against homologous sera (sera derived from the same patient as the parasite isolate tested) sampled at different time points. Figure 1a, b shows the kinetics of antibody acquisition. 3/6 patients developed antibodies to their baseline parasites (blue lines), with antibody acquisition rising over time (patients 119, 221 and 233). The other three made either a very weak, or no response at all i.e. patients 171,178 and 245 . This variation in antibody responses appeared not to be due to intrinsic differences in the ability of the parasites to be recognized by antibodies because there was no significant correlation between the homologous and heterologous recognition of these parasite isolates $(\mathrm{P}=0.221$, mixed-effects linear regression model) (Fig. 1c and Additional file 2: Figure S1), and there was no overall difference in antibodies to baseline vs. recurrent isolates measured in heterologous sera $(\mathrm{P}=0.215$, mixed-effects linear regression model). One explanation could be the duration of the infection (or more precisely: the infection with the clone or clones that were responsible for the malaria episode) before the patients presented to us. If diagnosed quickly and combined with the fast acting ACT used in this trial parasites could have been removed before an effective antibody response was mounted. This hypothesis could not be tested here.

\section{Recurrent Plasmodium falciparum infections tend to be} poorly recognized by pre-existing homologous antibodies As shown in Fig. 1a recognition of recurrent parasites by homologous sera taken earlier than the recurrence was generally poor. Figure $2 \mathrm{a}$ illustrates this further, where 

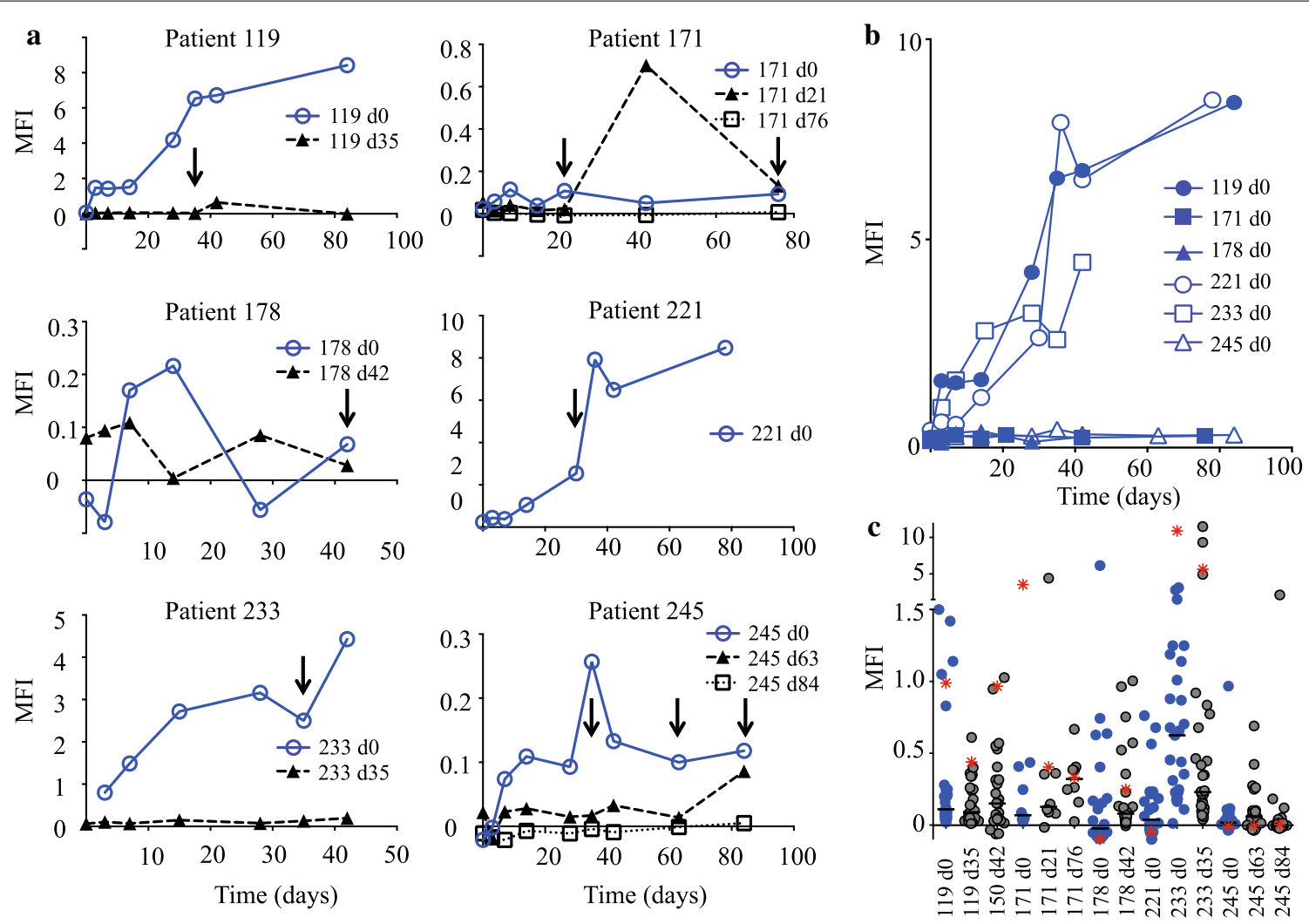

Fig. 1 Kinetics of antibody acquisition to baseline and recurrent isolates. a Surface recognition of individual parasite isolates by their corresponding homologous sera. Sera taken at different time-points after uptake of the child in the study were tested for recognition of the baseline and recurrent isolates, measured as mean fluorescence intensity (MFI). The recognition of baseline isolates is depicted in blue and that of recurrences in black. Arrows point to the time when the investigated recurrent parasitaemia was detected. $\mathbf{b}$ Homologous recognition of all baseline isolates is summarized to better illustrate the separation of responders versus non-responders. $\mathbf{c}$ Heterologous recognition of the individual isolates showing that isolates are variably recognized by heterologous sera, the median is shown as a horizontal bar. Hyperimmune serum recognition of the individual isolates is also shown (red asterisk)

homologous recognition of the individual recurrent isolates is shown and the day of recurrent parasitaemia is set at day 0 . To explore this further, this pre-existing homologous recognition of recurrent isolates was compared to recognition by a panel of heterologous day 0 (acute) and day 14 (convalescent) sera from other patients in the same study (Table 3). Recurrent parasites tended to be recognized less well by homologous sera than heterologous sera, although with only borderline significance (Fig. 2b) $\mathrm{P}=0.063$, mixed-effects linear regression model). Overall these observations are consistent with the idea that parasites express antigens corresponding to gaps in the repertoire of antibodies carried by the host.

\section{Genotyping}

All sera used to test heterologous recognition of the isolates described above were derived from patients who had also experienced a recurrent, i.e. secondary infection during follow-up. Those patients who were unable to clear their parasites in spite of treatment, resulting in a recrudescent infection are of specific interest. Therefore, MSP2 genotyping analysis was performed on the parasite DNA isolated from these patients to differentiate between recrudescence (persistent blood stage infection) and re-infection (new infection emerging from the liver). A recrudescent infection is defined by the presence of at least one genotype during follow-up also detected before treatment. Thus, $6 / 18$ patients whose sera were part of the heterologous serum panel were confirmed to have recrudescent infections within the follow-up period (Table 3).

\section{Patients carrying recrudescent infections tend to have a broader antibody repertoire than patients who cleared their infections}

Next, the anti-VSA antibody repertoires of the 6 patients with recrudescence was compared to the 12 patients who cleared their initial infections (Table 3). For this their ability to recognize the 14 parasite isolates from the 7 patients described above was measured (Table 2 and Additional 

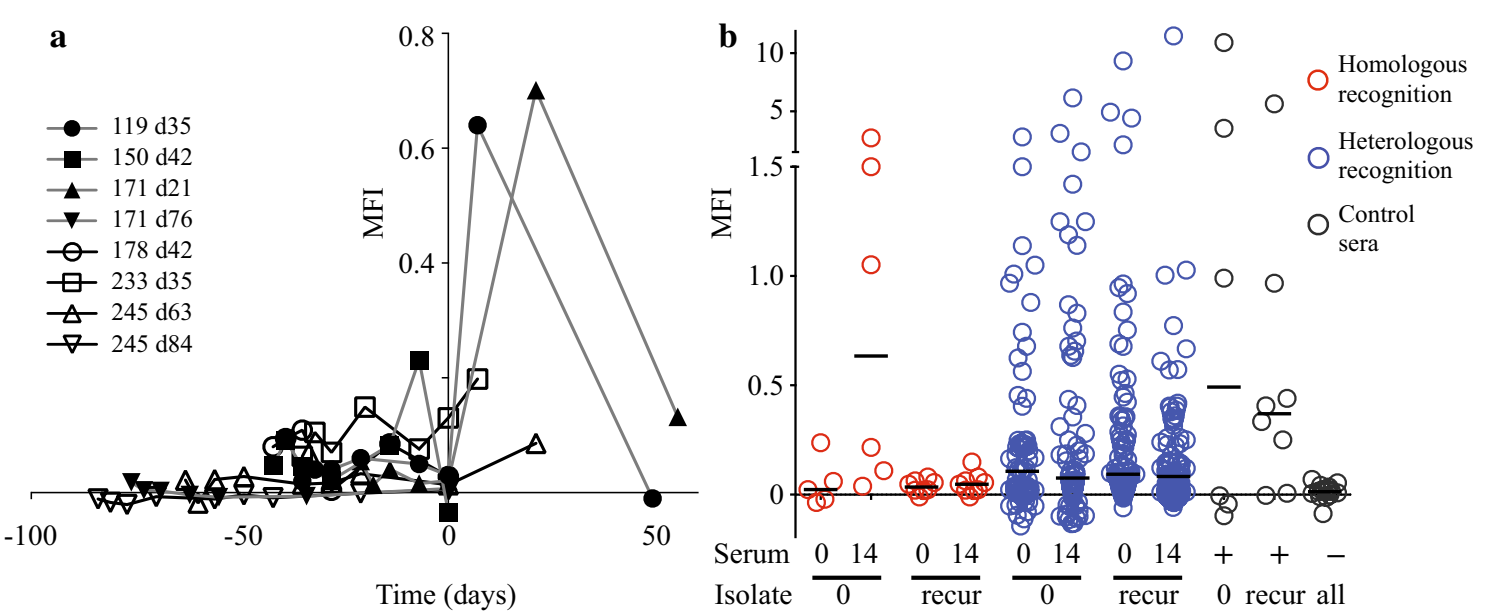

Fig. 2 Recurrent infections exploit gaps in antibody repertoire against surface of infected red blood cells. a Homologous surface recognition of individual recurrent isolates, where the day of each recurrent parasitaemia is set at day 0 . $\mathbf{b}$ Homologous recognition of all isolates as well as heterologous recognition is shown. Homologous recognition of the isolates is shown in red, and heterologous recognition of the same isolates is shown in blue. For comparison, recognition of all the isolates by hyperimmune and nonimmune sera is also shown, in gray. The sera used was taken at day 0 (at the uptake of the patient in the study), or at day 14 (convalescent sera), as indicated. The isolates were divided into baseline and recurrent isolates, as indicated below the graph. Homologous sera taken before recurrence recognizes the recurrent parasites less than their overall recognition by heterologous sera $(P=0.063)$. MFI: Mean fluorescence intensity

file 2: Figure S1). The anti-VSA antibody repertoire of the sera from children who carried recrudescent infections was higher than that of children with only reinfections (Fig. 3), although only borderline significant $\mathrm{P}=0.059$ as tested using a linear mixed effect regression model. To exclude that this is not simply an effect of reduced drug sensitivity of the parasite, existing data from all children in the initial study experiencing recurrences [17] was used to re-analyse risk of recrudescence as a function of the $\mathrm{IC}_{50}$ values for the long-acting drugs lumefantrine and piperaquine. A recrudescence is not determined by the sensitivity of the baseline isolate to the long-acting drugs used in the study (Additional file 3: Table S2). There was no indication of tolerance or resistance in the response to dihydroartemisinin in the study [17].

It is possible that submicroscopic infections (undetected microscopically but persisting until the recrudescence was detected), may themselves boost antibodies. However, the anti-VSA antibody repertoire in children who later had recrudescent parasites was higher than in those who later had recurrent infections at day 0 (Fig. 3). This suggests that sustained submicroscopic infection in children with recrudescences was not responsible for the difference in antibody levels between these two groups of children. One explanation for the difference is that the ability to recognize heterologous parasites promotes chronic asymptomatic infection as previously proposed by others $[9,23-25]$.

\section{Discussion}

Antibodies that bind parasite-encoded antigens on the surface of infected red blood cells (variant surface antigens, VSA) have been implicated in alleviating specific pathogenic interactions between parasite ligands and endothelial host receptors [26], but far less is known about the role of anti-VSA antibodies in benefiting parasite survival. From an evolutionary perspective, it is expected that natural selection favours parasite immune evasion strategies, including the display of VSAs, that increase survival in the form of asymptomatic maintenance of transmission reservoirs.

The transition from P. falciparum infections that trigger disease to asymptomatic infections is well documented in populations with high re-infection rates, but the exact nature of the host-parasite interactions that orchestrate this survival strategy of the parasite has been difficult to study, and recurrent $P$. falciparum infections in paediatric patients within an age bracket that encompasses the switch from symptomatic to asymptomatic infections could provide a useful readout.

Children who received treatment for malaria were recruited and were then followed-up to detect recurrence of infections. Stratification of recurrent infections in recrudescent primary infections or new secondary infections gave us a functional in vivo readout to assess the effects of anti-VSA responses determined at baseline. 


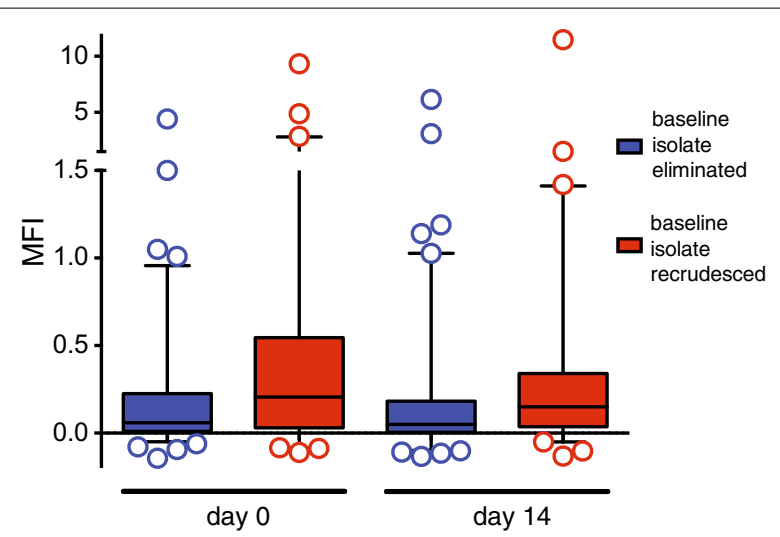

Fig. 3 Patients harbouring primary isolates that subsequently recrudesced have higher background recognition than patients that eliminated primary infections $(P=0.059)$. Shown here is the mean fluorescence intensity (MFI) of the heterologous recognition (of 14 isolates) by sera from 12 patients who eliminated their infections (and were later reinfected) (blue) compared to the 6 patients with recrudescences (red). The results are further divided into sera from day 0 (acute) and day 14 (convalescence) to show that the measured immune response is relatively stable, i.e. does not fluctuate from day to day, and is not due to submicroscopic infections still present in children with recrudescences in day 14 sera. Boxes show the interquartile range, whiskers the 5th-95th percentile and the median is shown as a horizontal bar. Single measurements outside the 5th95th percentile are shown individually

The pattern of antibody responses relating to malaria and recurrent infections were consistent with previous studies showing that episodes of disease are associated with specific gaps in the anti-VSA repertoire [14-16]. There was a tendency for antibodies in heterologous sera to recognize recurrent isolates to a higher degree than antibodies in day 14 sera recognized parasites from subsequent homologous recurrent isolates.

Persistent primary infections were detected as mild or asymptomatic recrudescences in patients with higher than average heterologous recognition capacity measured at day 0 . This is consistent with a potential role of anti-VSA antibodies maintaining chronic infections [9, 23-25]. Recker et al. have proposed a model in which a higher (transient) cross-reactive immune response can potentially slow down the development of long-lived variant specific responses, thereby prolonging the duration of infection [23-25].

A model in which anti-VSA antibodies help sustain chronic infections helps to explain previous observations of higher than average risk of recrudescent infections in young children $[3,27]$. This phenomenon in early childhood may be best explained by the immaturity of acquired immune mechanisms that directly contribute to the elimination of infections, and that maturity of these effector mechanisms may be a prerequisite for developing tolerance to subpatient, persistent infections.

This pilot study is limited by a small sample size. Recrudescences are rarely observed after highly efficacious artemisinin-combination therapy, and the definition of a recrudescent infection was based on a repeat polymorphism of a single, if highly variable, gene (MSP2). This method is reliable at a population level, but has limitations at the individual level because there will always be a bias toward majority genotypes. Studies in children also constrain the amount of sample volume, here only one measurement per isolate-serum pair tested was performed. Future larger studies will be required to confirm the hypotheses set out in this study.

It would be very interesting to make a detailed comparison of expressed VSA from recrudescent parasites in comparison with those that are isolated at day 0 . It was not possible to do this accurately in this study because blood for which white blood cells had not been removed was used. Standard conditions for DBL-alpha tag amplification cannot be used in the presence of high levels of human RNA. A preliminary analysis using amplification at higher temperature was performed and no major changes in the profile of var genes expressed at different time points was observed. Specific studies to analyse such differences in transcriptome between isogenic parasites at different points during the establishment of chronic infections would be of great value in understanding of how these infections become established.

\section{Conclusions}

In short, here data is presented that support the "hole in the wall" model, i.e. a framework for explaining how the parasite exploits gaps in the anti-VSA repertoire of the host. It is suggested that this model may be key to understanding how chronic infections are established in humans. First data is provided to support the hypothesis that a more complete anti-VSA antibody repertoire acquired after repeated infections could favour the transition from an uncomplicated malaria episode to an asymptomatic carrier status. These findings must be confirmed in larger studies, but it opens up to new interpretations of immunological data, and to the usage of recrudescence as an alternative, possibly more useful resource to measure immunity to malaria. 


\section{Additional files}

Additional file 1: Table S1. Samples and flow cytometry data used for analysis in this article.

Additional file 2: Figure S1. Heat map showing heterologous recognition of each individual isolate-serum pair (and homologous recognition highlighted by bold boxes). Mean fluorescence intensity is shown for each isolate-serum pair, and is colour graded in a scale from light green (lowest values), to dark green (highest values).

Additional file 3: Table S2. A recrudescence is not determined by the drug sensitivity of the baseline isolate.

\section{Abbreviations}

VSA: variant surface antigens; PfEMP1: Plasmodium falciparum Erythrocyte Membrane Protein 1; MSP2: merozoite surface protein 2; irbc: infected red blood cell; MFI: mean fluorescence intensity.

\section{Authors' contributions}

PS and JP conducted the initial study and collected samples under supervision of SB. Laboratory based work was performed by ES and AJ under supervision of SB and PB. Analysis of data was performed by EJP, ES and SB. ES, SB and PB wrote the manuscript. All authors read and approved the final manuscript.

\section{Author details}

${ }^{1}$ Kenya Medical Research Institute/Wellcome Trust Research Programme, Center for Geographic Medicine Research-Coast, P. O. Box 428, Kilifi 80108, Kenya. 2 Institute of Hygiene, University of Heidelberg School of Medicine, 69120 Heidelberg, Germany. ${ }^{3}$ International Livestock Research Institute, Old Naivasha Road, Nairobi, Kenya. ${ }^{4}$ Department of Infectious Diseases, Molecular Virology, University Hospital Heidelberg, Heidelberg, Germany. ${ }^{5}$ Department of Clinical Pharmacology, School of Medicine, Muhimbili University of Health and Allied Sciences, P.O. Box 65010, Dar es Salaam, Tanzania. ${ }^{6}$ German Center for Infection Research (DZIF), Wilhelmstraße 27,72074 Tübingen, Germany. ${ }^{7}$ Institute for Tropical Medicine, University of Tübingen, Tübingen, Germany. ${ }^{8}$ Nuffield Department of Medicine, Centre for Tropical Medicine, Oxford University, Oxford OX3 7LJ, UK. ${ }^{9}$ Department of Pathology, Cambridge University, Tennis Court Road, Cambridge CB2 1QP, UK. ${ }^{10}$ Present Address: International Livestock Research Institute, Old Naivasha Road, P.O. Box 30709, Nairobi 00100, Kenya.

\section{Acknowledgements}

We are indebted to the children and mothers who participated in the study. We would like to thank the Pingilikani Dispensary Health Committee for facilitating the study, the KEMRI-CGMRC Clinical Trials Team for their role in patient recruitment and the study nurses. We would also especially like to thank Oscar Kai for his assistance in the lab and George Warimwe for helpful comments. This work is published with the permission of the Director of KEMRI.

\section{Competing interests}

The authors declare that they have no competing interests.

\section{Availability of data and materials}

All data generated or analysed during this study are included in this published article and its additional files.

\section{Consent for publication}

Written consent for publication was provided by parent or guardian.

\section{Ethics approval and consent to participate}

The study was approved by the National KEMRI Ethical Review Committee (SSC 946), Kenya and the Ethics Committee, Heidelberg University School of Medicine, Germany and is registered with Controlled-Trials.com ISRCTN88705995. Parents or guardians provided written informed consent. IRB approvals was granted for the use of samples for the present analysis.

\section{Funding}

$E S, A R$, and SB were funded by a grant to SB (A7, SFB544, DFG), SB is currently funded by German Center for Infection Research (DZIF), TTU Malaria. PB was funded by Wellcome Trust Programme Grants 084535 and 092741 . The funding sources had no involvement in study design, in the collection and interpretation of data or in the writing of the report.

\section{Publisher's Note}

Springer Nature remains neutral with regard to jurisdictional claims in published maps and institutional affiliations.

Received: 17 July 2017 Accepted: 28 October 2017

Published online: 07 November 2017

References

1. Cohen S, Mc Gl, Carrington S. Gamma-globulin and acquired immunity to human malaria. Nature. 1961;192:733-7.

2. Sabchareon A, Burnouf T, Ouattara D, Attanath P, Bouharoun-Tayoun $\mathrm{H}$, Chantavanich $\mathrm{P}$, et al. Parasitologic and clinical human response to immunoglobulin administration in falciparum malaria. Am J Trop Med Hyg. 1991;45:297-308.

3. Borrmann S, Matsiegui PB, Missinou MA, Kremsner PG. Effects of Plasmodium falciparum parasite population size and patient age on early and late parasitological outcomes of antimalarial treatment in children. Antimicrob Agents Chemother. 2008:52:1799-805.

4. Bull PC, Lowe BS, Kortok M, Molyneux CS, Newbold Cl, Marsh K. Parasite antigens on the infected red cell surface are targets for naturally acquired immunity to malaria. Nat Med. 1998;4:358-60.

5. Giha HA, Staalsoe T, Dodoo D, Roper C, Satti GM, Arnot DE, et al. Antibodies to variable Plasmodium falciparum-infected erythrocyte surface antigens are associated with protection from novel malaria infections Immunol Lett. 2000;71:117-26.

6. Marsh K, Otoo L, Hayes RJ, Carson DC, Greenwood BM. Antibodies to blood stage antigens of Plasmodium falciparum in rural Gambians and their relation to protection against infection. Trans $\mathrm{R}$ Soc Trop Med Hyg. 1989:83:293-303.

7. Dodoo D, Staalsoe T, Giha H, Kurtzhals JA, Akanmori BD, Koram K, et al. Antibodies to variant antigens on the surfaces of infected erythrocytes are associated with protection from malaria in Ghanaian children. Infect Immun. 2001;69:3713-8.

8. Ofori MF, Dodoo D, Staalsoe T, Kurtzhals JA, Koram K, Theander TG, et al. Malaria-induced acquisition of antibodies to Plasmodium falciparum variant surface antigens. Infect Immun. 2002;70:2982-8.

9. Warimwe GM, Recker M, Kiragu EW, Buckee CO, Wambua J, Musyoki $\mathrm{JN}$, et al. Plasmodium falciparum var gene expression homogeneity as a marker of the host-parasite relationship under different levels of naturally acquired immunity to malaria. PLOS ONE. 2013;8:e70467.

10. Staalsoe T, Hamad AA, Hviid L, Elhassan IM, Arnot DE, Theander TG. In vivo switching between variant surface antigens in human Plasmodium falciparum infection. J Infect Dis. 2002;186:719-22.

11. Chan JA, Howell KB, Reiling $L$, Ataide R, Mackintosh CL, Fowkes FJ, et al. Targets of antibodies against Plasmodium falciparum-infected erythrocytes in malaria immunity. J Clin Invest. 2012;122:3227-38.

12. Scherf A, Lopez-Rubio JJ, Riviere L. Antigenic variation in Plasmodium falciparum. Annu Rev Microbiol. 2008;62:445-70.

13. Rottmann M, Lavstsen T, Mugasa JP, Kaestli M, Jensen AT, Muller D, et al. Differential expression of var gene groups is associated with morbidity caused by Plasmodium falciparum infection in Tanzanian children. Infect Immun. 2006;74:3904-11.

14. Warimwe GM, Keane TM, Fegan G, Musyoki JN, Newton CR, Pain A, et al. Plasmodium falciparum var gene expression is modified by host immunity. Proc Natl Acad Sci USA. 2009;106:21801-6.

15. Bull PC, Berriman M, Kyes S, Quail MA, Hall N, Kortok MM, et al. Plasmodium falciparum variant surface antigen expression patterns during malaria. PLoS Pathog. 2005;1:e26.

16. Warimwe GM, Fegan G, Musyoki JN, Newton CR, Opiyo M, Githinji $\mathrm{G}$, et al. Prognostic indicators of life-threatening malaria are associated with distinct parasite variant antigen profiles. Sci Transl Med. 2012;4:129ra145. 
17. Borrmann S, Sasi P, Mwai L, Bashraheil M, Abdallah A, Muriithi S, et al. Declining responsiveness of Plasmodium falciparum infections to artemisinin-based combination treatments on the Kenyan coast. PLoS ONE. 2011;6:e26005

18. Felger I, Tavul L, Beck HP. Plasmodium falciparum: a rapid technique for genotyping the merozoite surface protein 2. Exp Parasitol. 1993;77:372-5

19. Falk N, Maire N, Sama W, Owusu-Agyei S, Smith T, Beck HP, et al. Comparison of PCR-RFLP and Genescan-based genotyping for analyzing infection dynamics of Plasmodium falciparum. Am J Trop Med Hyg. 2006;74:944-50.

20. Trager $W$, Jensen JB. Human malaria parasites in continuous culture. Science. 1976;193:673-5

21. Bejon P, Warimwe G, Mackintosh CL, Mackinnon MJ, Kinyanjui SM, Musyoki JN, et al. Analysis of immunity to febrile malaria in children that distinguishes immunity from lack of exposure. Infect Immun. 2009;77:1917-23.

22. Payne RW, Harding S. A guide to REML in GenStat ${ }^{\circledR}$. 16th ed. Hemel Hempstead: VSN International; 2013
23. Recker M, Buckee CO, Serazin A, Kyes S, Pinches R, Christodoulou Z, et al. Antigenic variation in Plasmodium falciparum malaria involves a highly structured switching pattern. PLoS Pathog. 2011;7:e1001306.

24. Recker M, Gupta S. Conflicting immune responses can prolong the length of infection in Plasmodium falciparum malaria. Bull Math Biol. 2006;68:821-35.

25. Recker M, Nee S, Bull PC, Kinyanjui S, Marsh K, Newbold C, et al. Transient cross-reactive immune responses can orchestrate antigenic variation in malaria. Nature. 2004:429:555-8.

26. Turner L, Lavstsen T, Berger SS, Wang CW, Petersen JE, Avril M, et al. Severe malaria is associated with parasite binding to endothelial protein C receptor. Nature. 2013;498:502-5.

27. Djimde AA, Doumbo OK, Traore O, Guindo AB, Kayentao K, Diourte Y, et al Clearance of drug-resistant parasites as a model for protective immunity in Plasmodium falciparum malaria. Am J Trop Med Hyg. 2003;69:558-63.

\section{Submit your next manuscript to BioMed Central and we will help you at every step:}

- We accept pre-submission inquiries

- Our selector tool helps you to find the most relevant journal

- We provide round the clock customer support

- Convenient online submission

- Thorough peer review

- Inclusion in PubMed and all major indexing services

- Maximum visibility for your research

Submit your manuscript at www.biomedcentral.com/submit 